\title{
What is a plasmablast? A commentary to: "Plasmablastic lymphoma versus diffuse large B-cell lymphoma with plasmablastic differentiation: proposal for a novel diagnostic scoring system" by Sonja Catharina Boy et al., J. Hematopathol 2015
}

\author{
Olga Balagué • Antonio Martínez
}

Published online: 15 March 2015

(C) Springer-Verlag Berlin Heidelberg 2015

\begin{abstract}
Plasmablastic lymphoma is an aggressive neoplasm occurring in a sharply defined clinical setting, although other B cell lymphomas may show plasmacytic/plasmablastic differentiation. Here, we discuss the controversies in definition and nomenclature of these entities.
\end{abstract}

Keywords Plasmablastic lymphoma · HIV · EBER · Diffuse large B-cell lymphoma · Plasmablast · B-cell differentiation

Plasma cell differentiation is a terminal differentiation process orchestrated by the upregulation of transcription factors involved in secretory transformation, i.e. endoplasmic reticulum growth and immunoglobulin secretion, together with the downregulation of genes related to the germinal centre programme and maintenance of the B-cell phenotype. This complex process promotes morphological and phenotypical changes that are allied in normal B-cell development. However, secretory and plasma cell differentiation are different events and do not always go together.

Plasma cell differentiation with cytoplasmic expression of immunoglobulin has been described in almost every mature B-cell lymphoma category. Contrary to membrane-bound immunoglobulin, cytoplasmic immunoglobulin accumulates in the endoplasmic reticulum (ER) and Golgi to be secreted. Strong cytoplasmic immunoglobulin secretion is a feature of plasma cell neoplasms but can be also observed in B-cell

O. Balagué $(\bowtie) \cdot$ A. Martínez

Department of Pathology, Hospital Clinic, Barcelona, Spain e-mail: OBALAGUE@clinic.ub.es lymphomas of any type. Moreover, different clinical consequences of plasma cell differentiation may be observed in Bcell lymphomas. In low-grade B-cell lymphomas, the presence of plasma cell differentiation does not seem to affect the tumour behaviour but may be related to response to treatment targeting ER-dependent secretory pathways such as proteosome inhibitors in mantle cell lymphoma; in aggressive B-cell lymphomas, however, plasma cell differentiation is associated with a more aggressive behaviour and poor response to conventional treatments, plasmablastic lymphomas being the best example.

The last steps involved in plasma cell differentiation still have to be elucidated; therefore, the classification of neoplasms originated at this stage of maturation remains confusing with many overlapping terminologies and entities. Especially the term plasmablastic differentiation has been easily used in different scenarios leading to confusion and misunderstanding.

In immunology, a "plasmablast" refers to a short-lived differentiation stage between a post germinal centre B-cell and a mature plasma cell. Plasmablasts retain a proliferative capability together with an almost fully mature plasma cell phenotype [1]. For a hematologic cytologist, a plasmablast is the circulating tumour cell observed in peripheral blood smears from a patient with plasma cell myeloma. More recently, Isaacson used the term plasmablast for the HHV-8/CD20+ immunoblast-like cells found in the mantle zone of multicentric Castleman disease follicles and suggested that these give rise to the so-called plasmablastic lymphomas arising in this disease.

Plasmablastic lymphoma (PBL) was first described in 1997 [2] in a very specific clinical setting related to an immunodeficiency associated with HIV infection. However, in the last 
years, the clinical spectrum of the plasmablastic lymphoma became broader. In addition, some degree of plasmablastic differentiation can be observed in other entities including also some diffuse large B-cell lymphoma (DLBCL) that do not fulfil the criteria to be called plasmablastic lymphoma.

Plasmablastic differentiation is frequently observed in lymphoproliferative disorders related to immunosuppression (in the elderly, HIV-associated, post-transplantation) and associated with well-known oncogenic viruses such as Epstein-Barr virus (EBV) and HHV-8, chronic inflammation or anaplastic lymphoma kinase (ALK) translocations that have been shown to promote plasma cell phenotype and secretory differentiation (plasmablastic lymphoma, ALK + DLBCL, primary effusion lymphoma).

In 2004, Colomo et al. [3] described two subtypes of PBL. One group presented with a monotonous immunoblastic proliferation with a plasma cell phenotype, presenting in the oral mucosa or other extranodal localization in $\mathrm{HIV}+$ patients and with very frequent EBV infection. The second group showed nodal presentation, heterogeneous morphology with different degrees of plasmacytic differentiation and less often EBV association.

It is therefore not clear when "plasmablastic differentiation" is used what is meant: phenotypical differentiation: large cells with a terminal B-cell differentiation profile (CD20- PAX5- /CD138+Blimp1+ XBP1s+), morphological differentiation (plasmablastic myeloma, DLBL with plasmablastic features) or a specific entity (PBL oral cavity, PBL in Castleman disease/HHV-8 associated).

With the appearance of new immunohistochemical markers for transcription factors involved in the terminal Bcell differentiation process, different attempts have been done to establish a phenotype able to characterize PBL in order to segregate them from the CD20- DLBCL with plasmablastic features. None of the proposed markers on its own is able to sharpen the outlines of the entity; nevertheless, a combination of markers can be used. Montes-Moreno et al. [4] proposed negativity for CD20 and PAX-5 and expression of Blimp-1 and XBP-1s. Although Blimp-1 expression has been observed in DLBCL, the combination with XBP-1s expression seems very specific for PBL. Xbp-1 expression has been detected in germinal centre B-cells committed to plasma cell differentiation, and its expression is mutually exclusive with Pax-5 and $\mathrm{Bcl}-6$ expression. In tumours, XBP-1s is mainly expressed in aggressive lymphomas and in a large cell subpopulation in chronic lymphocytic leukaemia, even in the lack of overt morphological plasma cell differentiation, stressing the fact that secretory and plasma cell differentiation do not always walk together. Xbp-1s expression in the absence of Blimp-1 expression occurs very rarely [5]. The absence of B cell markers alone is not sufficient to distinguish DLBCL with plasmablastic differentiation from plasmablastic lymphoma. From a clinical point of view, there is also evidence that plasmablastic differentiation in the absence of a fully developed plasmablastic phenotype can confer worse prognosis to the patient. In that sense, establishing a border is maybe less important than understanding which factors are implicated in the poor prognosis of these neoplasmas.

Therefore, in our opinion, we should keep the term PBL to those cases with the full plasmablastic phenotype in the proper clinical setting. The better understanding of the physiological steps of the plasma cell differentiation will help to segregate all the entities that are know included under the umbrella of the plasmablast.

\section{References}

1. Tarte K, Zhan F, De Vos J, Klein B, Shaughnessy J Jr (2003) Gene expression profiling of plasma cells and plasmablasts: toward a better understanding of the late stages of B-cell differentiation. Blood 102: $592-600$

2. Delecluse HJ, Anagnostopoulos I, Dallenbach F, Hummel M, Marafioti T, Schneider U, Huhn D, Schmidt-Westhausen A, Reichart PA, Gross U, Stein H (1997) Plasmablastic lymphomas of the oral cavity: a new entity associated with the human immunodeficiency virus infection. Blood 89:1413-1420

3. Colomo L, Loong F, Rives S, Pittaluga S, Martínez A, LópezGuillermo A, Ojanguren J, Romagosa V, Jaffe ES, Campo E (2004) Diffuse large B-cell lymphomas with plasmablastic differentiation represent a heterogeneous group of disease entities. Am J Surg Pathol 28: 736-747

4. Montes-Moreno S, Gonzalez-Medina AR, Rodriguez-Pinilla SM, Maestre L, Sanchez-Verde L, Roncador G, Mollejo M, García JF, Menarguez J, Montalbán C, Ruiz-Marcellan MC, Conde E, Piris MA (2010) Aggressive large B-cell lymphoma with plasma cell differentiation: immunohistochemical characterization of plasmablastic lymphoma and diffuse large B-cell lymphoma with partial plasmablastic phenotype. Haematologica 95:1342-1349

5. Balague O, Mozos A, Martinez D, Hernandez L, Colomo L, Mate JL, Teruya-Feldstein J, Lin O, Campo E, LopezGuillermo A, Martinez A (2009) Activation of the endoplasmic reticulum stress-associated transcription factor $\mathrm{x}$ box-binding protein-1 occurs in a subset of normal germinal-center B cells and in aggressive B-cell lymphomas with prognostic implications. Am J Pathol 174:2337-2346 